Please do not remove this page

RMIT

UNIVERSITY

\title{
Liquid metal marbles
}

Sivan, Vijay Prasad; Tang, Shiyang; O'Mullane, Anthony; Petersen, Phred; Eshtiaghi, Nicky; Kalantar Zadeh, Kourosh; Mitchell, Arnan

https://researchrepository.rmit.edu.au/esploro/outputs/9921859311801341/filesAndLinks?institution=61RMIT_INST\&index=null

Sivan, V. P., Tang, S., O’Mullane, A., Petersen, P., Eshtiaghi, N., Kalantar Zadeh, K., \& Mitchell, A. (2013).

Liquid metal marbles. Advanced Functional Materials, 23(2), 144-152.

https://doi.org/10.1002/adfm.201200837

Document Version: Accepted Manuscript

Published Version: https://doi.org/10.1002/adfm.201200837

Repository homepage: https://researchrepository.rmit.edu.au

(c) 2013 WILEY-VCH Verlag GmbH \& Co. KGaA, Weinheim.

Downloaded On 2023/04/26 21:37:38 +1000

Please do not remove this page 
Thank you for downloading this document from the RMIT Research Repository.

The RMIT Research Repository is an open access database showcasing the research outputs of RMIT University researchers.

RMIT Research Repository: http://researchbank.rmit.edu.aul

\section{Citation:}

Sivan, V, Tang, S, O'Mullane, A, Petersen, P, Eshtiaghi, N, Kalantar Zadeh, K and Mitchell, A 2013, 'Liquid metal marbles', Advanced Functional Materials, vol. 23, no. 2, pp. 144-152.

See this record in the RMIT Research Repository at:

https://researchbank.rmit.edu.au/view/rmit:20460

Version: Accepted Manuscript

\section{Copyright Statement:}

(c) 2013 WILEY-VCH Verlag GmbH \& Co. KGaA, Weinheim.

Link to Published Version:

http://dx.doi.org/10.1002/adfm.201200837 
DOI: $10.1002 / \mathrm{adfm}$

Submitted to

\section{Liquid Metal Marbles}

By V. Sivan, S. Y. Tang, A. P. O’Mullane, P. Petersen, N. Eshtiaghi, K. Kalantar-zadeh* and

A. Mitchell*

Dr. V. Sivan, Mr. S. Y. Tang and Assoc. Prof. K. Kalantar-zadeh*

School of Electrical and Computer Engineering, RMIT University, GPO Box 2476,

Melbourne VIC 3001 (Australia)

* kourosh.kalantar@,rmit.edu.au

Dr. A. P. O’Mullane

School of Applied Science, RMIT University, GPO Box 2476, Melbourne VIC 3001 (Australia)

Mr. P. Petersen

School of Media and Communication, RMIT University, GPO Box 2476, Melbourne VIC 3001 (Australia)

Dr N. Eshtiaghi

School of Civil, Environmental and Chemical Engineering, RMIT University, GPO Box 2476, Melbourne VIC 3001 (Australia)

Prof. A. Mitchell**

Centre for Ultra-high bandwidth Devices for Optical Systems (CUDOS), School of Electrical and Computer Engineering, RMIT University, GPO Box 2476, Melbourne VIC 3001

(Australia)

**arnan.mitchell@,rmit.edu.au

Keywords: Liquid metal; liquid marble; galinstan; soft electronics; metal-semiconductormetal; electrochemical; heavy metal ion sensor.

We introduce "liquid metal marbles" which are droplets of liquid metal encapsulated by micro- or nano-particles. We coat droplets of galinstan liquid metal with insulators (including Teflon and silica), and also semiconductors (including $\mathrm{WO}_{3}, \mathrm{TiO}_{2}, \mathrm{MoO}_{3}, \mathrm{In}_{2} \mathrm{O}_{3}$ and carbon nanotubes) by rolling over a powder bed and also by submerging in colloidal suspensions. We show that these marbles can be split and merged, can be suspended on water and are even stable when moving under the force of gravity and impacting a flat solid surface. Further, we show the marble coating can operate as an active electronic junction; and that the nanomaterial coated liquid metal marble can act as a highly sensitive electrochemical based heavy 
Submitted to

metal ion sensor. This new element thus represents a significant platform for the advancement of research into soft electronics.

\section{Introduction}

The term 'liquid marble' was first introduced nearly a decade ago to describe droplets of aqueous fluid encapsulated within hydrophobic particles. ${ }^{[1]}$ These liquid marbles behave, to some degree, like solid particles, but due to the fact that their structural form is dominated by surface tension, they exhibit a number of unique properties, including very small contact area with surfaces leading to low friction rolling, superhydrophobic interactions with other fluids and the ability to be split or fused together with self-healing encapsulation layers. ${ }^{[2]}$ Liquid marbles rely on surface tension and can be formed with both non-polar or polar fluids including water. ${ }^{[1-5]}$ Indeed, water solutions encased with hydrophobic particles have great potential for encapsulating biological environments even at nanolitre scales. ${ }^{[6]}$ Due to the unique capabilities of liquid marbles, there has been a large increase in the number of studies on their properties as well as their practical applications. ${ }^{[7-10]}$

Liquid metals have a long history of study in the fields of analytical chemistry, electronics, and experimental physics. Traditionally, the most common liquid metal to be studied has been mercury. ${ }^{[1]}$ The toxicity of mercury however rendered applications based on this liquid metal unattractive and thus research interest into the applications of liquid mercury has remained niche. Recently, less hazardous liquid metals including eutectic alloys of gallium (75\%) and indium $(25 \%)(\mathrm{eGaIn}){ }^{[12,13]}$ and of gallium $(68.5 \%)$, indium $(21.5 \%)$ and tin $(10 \%)$, (Galinstan) $)^{[11,14,15]}$ have become readily available and thus research interest in these alternative liquid metals is gaining momentum. In general, liquid metals offer several unique properties including high density $\left(6440 \quad \mathrm{~kg} / \mathrm{m}^{3}{ }^{[11]}\right)$, high surface tension 


\section{Submitted to}

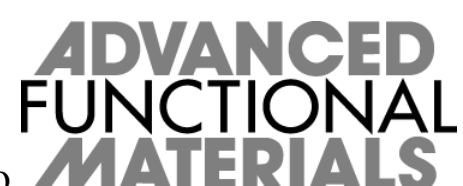

$\left(534.6 \pm 10.7 \mathrm{mN} / \mathrm{m}^{[14]}\right)$ and extremely low vapor pressure $\left(<10^{-6} \mathrm{~Pa}\right.$ at $\left.500^{\circ} \mathrm{C}^{[11]}\right)$ allowing them to operate as liquids in vacuum conditions and high temperatures. ${ }^{[1]}$ Most importantly, liquid metals offer the highest conductivity of any liquids, with orders of magnitude less conducting loss than ionic fluids making them attractive for various applications, such as in soft electronic components, $\left.{ }^{[12,}{ }^{13}\right]$ stretchable antennas, ${ }^{[16-18]}$ interconnects, ${ }^{[19,}$ 20] electromagnets, ${ }^{[21]}$ MEMS switches ${ }^{[22]}$ and reconfigurable wires. ${ }^{[23]}$

Despite these many desirable properties, liquid metals share one major disadvantage for practical use in that they are highly corrosive, particularly to other metals. ${ }^{[24]}$ This means that they will dissolve and amalgamate with solid contact metals such as gold which would be typically used to electrically interface to the liquid metal to exploit its properties. Liquid metals based on eutectic alloys of gallium in ambient air, forms a thin oxide layer which will also adhere strongly to ionic surfaces such as glass ${ }^{[11,14,25]}$ and even relatively low surface energy polymers including polydimethylsiloxane (PDMS) which is often used to manipulate fluids. ${ }^{[22]}$ In order to effectively exploit the attractive properties of liquid metals, a means must be found to prevent the fluid metal from adhering to its environment or corroding its metal contacts, while maintaining both the flexible re-configurability offered by the fluid and access to the electronic properties of the liquid metal.

In this paper, we present a novel approach to the use of liquid metals through the creation of 'liquid metal marbles'. Here we encapsulate small droplets of galinstan within a coating of nanoscale powders. We comprehensively studied the physical properties of such liquid metal marbles in terms of their contact angle, splitting and fusing upon applying a force, floating on aqueous media and dynamic properties during their free fall and impact on hydrophobic and hydrophilic surfaces. We show that powders of nanoparticles can be used to achieve metal liquid marbles with semiconducting properties at their surface. Achieving this, we demonstrate several liquid metal marble properties including, the capability for forming 
Submitted to

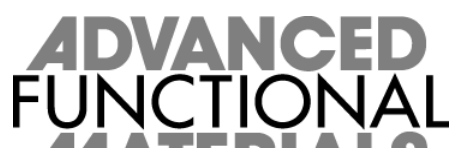

metal-semiconductor-metal (MSM) soft electronic devices and investigate their $I-V$ transfer characteristics. Finally, we demonstrate the utility of this new metal liquid marble through its use as a highly sensitive and selective heavy metal ion sensor.

\section{Realization of liquid metal marbles}

Liquid marbles are typically realized by rolling a small aqueous droplet over a bed of powder to achieve a uniform coating. ${ }^{[1]}$ For aqueous droplets, the powder coating must be hydrophobic, such that the surface tension prevents the particles from sinking into the fluid. ${ }^{[1]}$ Liquid metals tend to wet most materials to some extent; however, the exceptionally strong cohesive forces between the alloyed liquid metal elements results in a contact angle of greater than $90^{\circ}$ being observed when liquid metal droplets rest on almost all non-metallic material surfaces. Thus, most non-metals are considered as 'phobic' to the liquid metal. This greatly expands the range of powder materials that do not sink or amalgamate into the liquid metal and can be used as liquid marble coatings.

In this paper, we report on the realisation and application of liquid marbles formed from the liquid metal galinstan, (a eutectic alloy of $\mathrm{Ga}$, In and $\mathrm{Sn}$ ). We adapted two methods for coating the liquid metal with powder: (a) rolling the droplet on a powder bed, and (b) submerging the liquid metal droplet in a colloidal suspension of micro- or nano-partices.

Figure 1 present Scanning Electron Microscope (SEM) images of liquid metal marbles formed using these techniques. Figure $1 \mathrm{a}$ and $1 \mathrm{~b}$ present images of liquid metal marbles formed by rolling a droplet of galinstan on a powder bed of $\mathrm{WO}_{3}$ nanoparticles (particle size ca. $80 \mathrm{~nm})$. Here it can be seen that the coating is not uniform and can consist of multiple layers of particles. Tears evident in the coating are due to the expansion of the liquid marble when imaged under vacuum in the SEM. In a traditional liquid marble, such tears would 
Submitted to

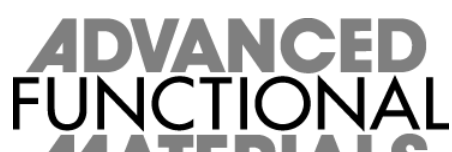

'heal' due to the redistribution of the particles on the surface. It has been previously reported that any exposed region of the galinstan alloy to air or even as little as $0.2 \%$ volume of oxygen will cause a thin, solid film of gallium oxide to form at the surface. ${ }^{[26]}$ We believe that such a film may be present on the galinstan marbles which would prevent the free movement of particles on the surface. This could explain the tears observed in Figures 1a.

Figure 1c shows the surface of a liquid marble formed by submerging a galinstan droplet in a suspension of the same $80 \mathrm{~nm} \mathrm{WO}_{3}$ nanoparticles in DI water $\left(0.7 \mathrm{~g} \mathrm{WO}_{3}: 50 \mathrm{ml}\right.$ DI water $)$. A sparse, but uniform coating is observed. If the concentration of particles in suspension is increased, the density of the coating can also be increased (Supplementary S1), demonstrating that submersion in a suspension allows controllability in the loading of the particles on the surface. A statistical plot of the surface area to particle coverage is provided in Supplementary $\mathrm{S} 2$. The sparsely coated marbles could be rolled on a normally wetting surface (such as $\mathrm{Si}$ ) without it adhering to the surface (Supplementary S3). We believe that this is the first reported use of immersion in a colloidal suspension to form liquid marbles and further anticipate that this technique could be extended to the encapsulation of any immiscible droplets in aqueous media.

Our observations have shown that suitable powders include typical low-surface energy materials such as Teflon, but extend to polar insulators such as $\mathrm{SiO}_{2}$ and even semiconductors such as $\mathrm{ZnO}$ and $\mathrm{WO}_{3}$ and conducting carbon nanotubes. The SEM images of surface coverage with some of these powder materials is presented in Figure 2. 


\section{Submitted to}
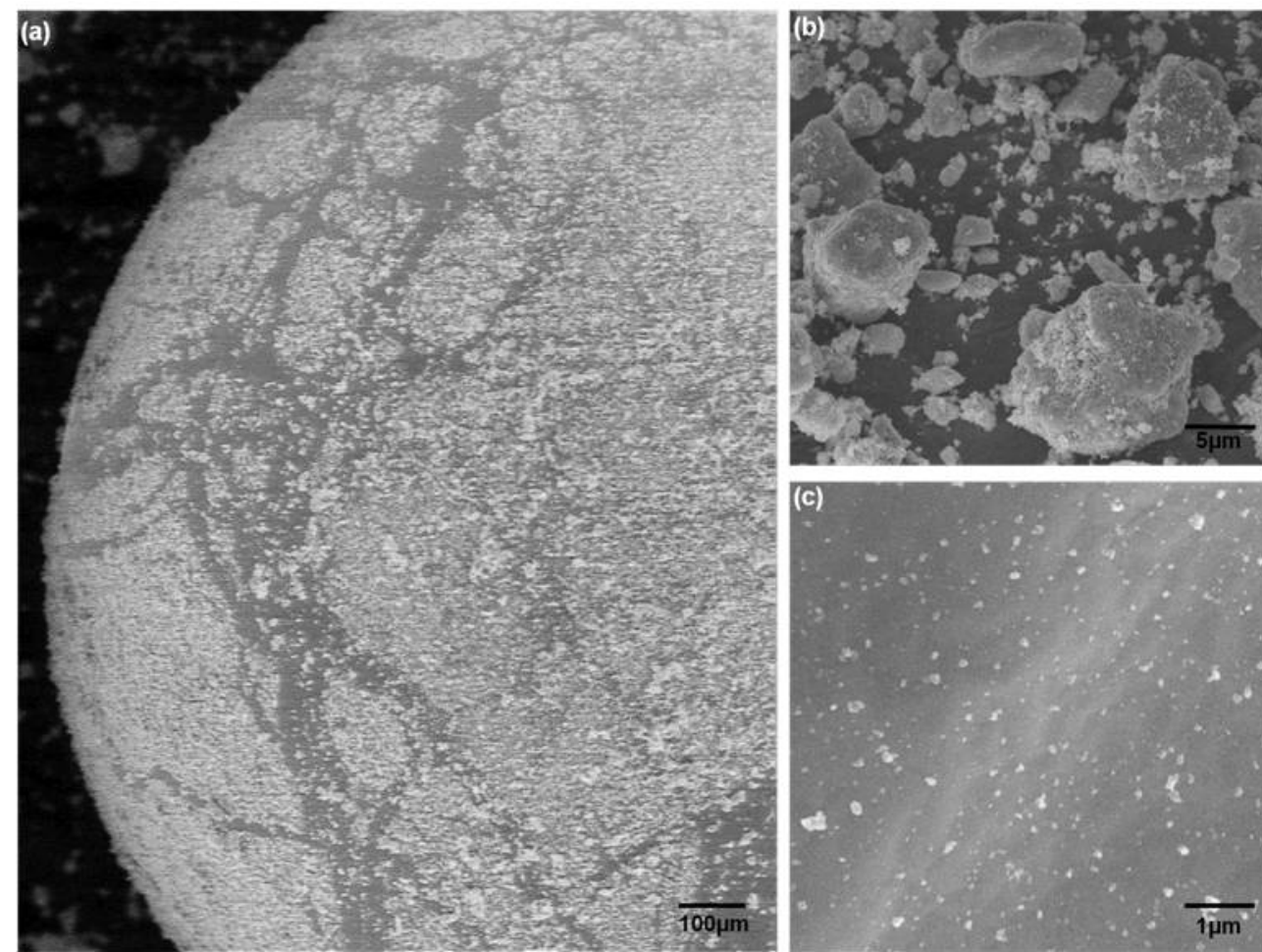

Figure 1: Scanning electron microscope image of a liquid metal marble, $\boldsymbol{a}, \boldsymbol{b})$ galinstan droplet encapsulated in a coating of 80nm $\mathrm{WO}_{3}$ nanoparticles by rolling it on a powder bed, c) surface of the liquid metal marble from by submerging a liquid metal droplet in a colloidal dispersion of $0.1 \mathrm{~g}$ of $\mathrm{WO}_{3}$ nanoparticles in $50 \mathrm{ml}$ DI water,
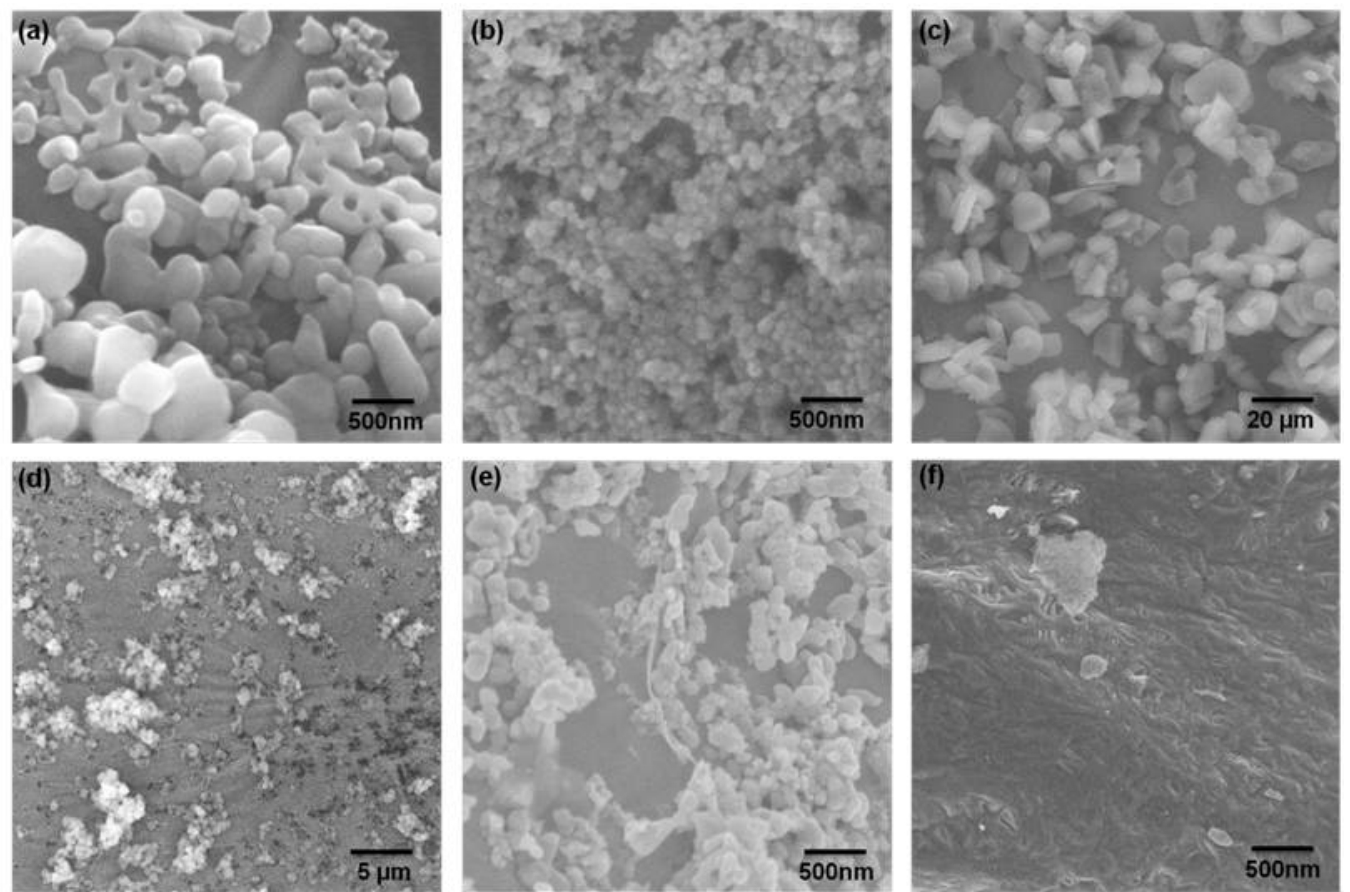

Figure 2: Scanning electron microscope (SEM) image of different powders that are coated on the surface of galinstan by rolling on a powder bed: (a) ZnO nanoparticles( 100nm),

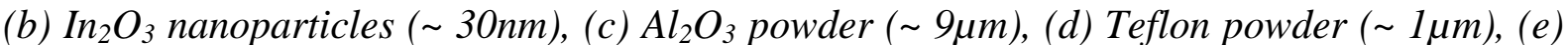
$\mathrm{TiO}_{2}$ nanoparticles $(\sim 100 \mathrm{~nm})$ and $(f)$ Single wall carbon nanotubes $(\sim 1 \mathrm{~nm}$ diameter and lengths of several micrometers) 


\section{Physical properties}

Submitted to

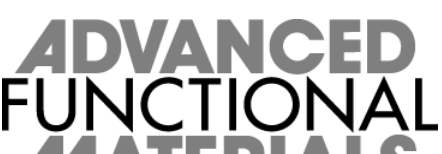

MATERIALS

Liquid marbles are attractive due to their many unique physical properties, which include a large contact angle and hence low rolling friction; the ability to be split into smaller marbles or merged together; the ability to rest on the surface of various liquids supported by surface tension; and the ability to remain stable under impact. ${ }^{[2]}$

Galinstan has the property to form a thin oxide layer when exposed to air, which may affect the true nature of this material. We reduce the oxide layer by treating it with diluted $\mathrm{HCl}$ solution $(6 \%),{ }^{[27]}$ before coating the droplet with the powder. In the following we report on a number of investigations to determine whether liquid metal marbles also possess such properties.

Firstly, in Figure 3a we present the shape of the galinstan droplet with the naturally formed oxide layer resting on wetting (silicon) and non-wetting (Teflon) surfaces. The tips formed from the dispensing of the droplet can be observed. This is attributed to the oxidation of the surface of galinstan. ${ }^{[14]}$ When the droplets were treated with diluted $\mathrm{HCl}$ solution to reduce the oxide layer, the droplets become less viscous and the tip conforms into a spherical shape as were previously reported. ${ }^{[27]}$ Lastly, we present the coated (rolled on $80 \mathrm{~nm} \mathrm{WO}_{3}$ powder bed) droplets of galinstan resting on wetting and non-wetting surfaces. The coated droplets possess the characteristics similar to those of liquid marbles by not wetting the surface.

To test the ability to merge and split the liquid metal marbles, we formed two marbles by rolling galinstan droplets on $80 \mathrm{~nm} \mathrm{WO}_{3}$ powder bed, placed them in close proximity and mechanically pressed them together. Figure $3 \mathbf{b}$ presents a photograph of the original separate droplets, the application of a spatula to press the marbles together, and the resulting fused marble. The final marble is relatively large and are quite spherical in shape. To demonstrate splitting, a single liquid marble was formed by rolling on a $80 \mathrm{~nm} \mathrm{WO}_{3}$ powder bed and then 


\section{FUNCTIONAL}

was cut using a scalpel. Figure 3c presents photographs of the original marble, cutting with a scalpel blade and the resulting pair of marbles.

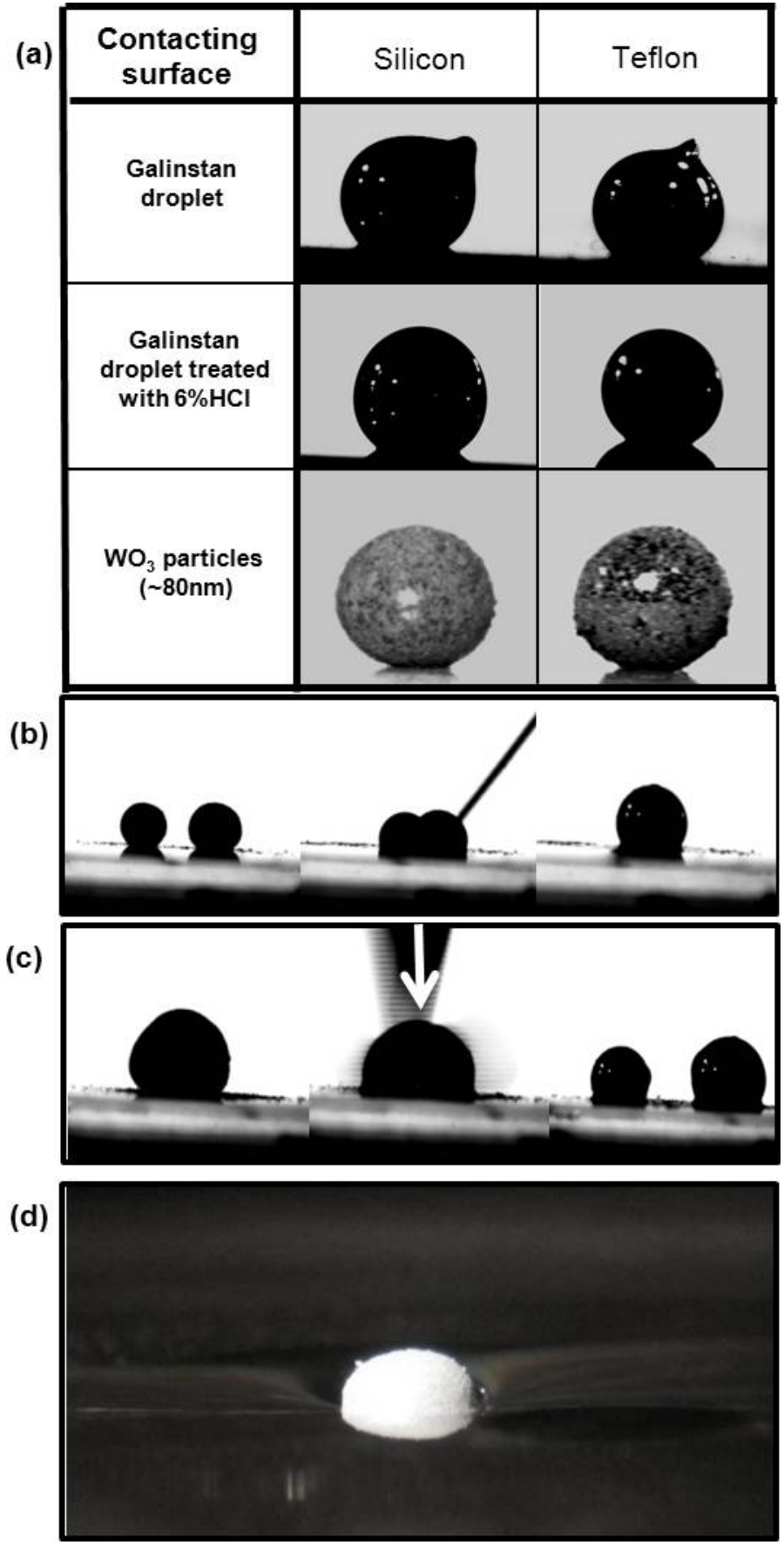

Figure 3: Physical properties of liquid metal marbles - a) Images of a galinstan droplet with naturally formed native oxide layer in ambient air, a galinstan droplet treated with diluted $\mathrm{HCl}$ and $\mathrm{WO}_{3}$ powder coated galinstan on a wetting (Si) and non-wetting (Teflon) surface. $W_{3}$ nanoparticles was used to coat the galinstan droplet by rolling it on a powder bed. $\left.\boldsymbol{b}\right)$ 
Submitted to

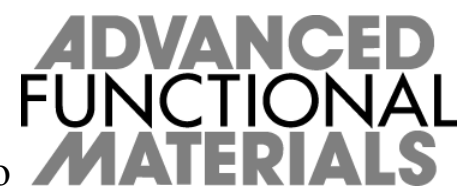

Merging of two dilute $\mathrm{HCl}$ treated $\mathrm{WO}_{3}$ powder $(\sim 80 \mathrm{~nm})$ coated galinstan droplets. $\mathrm{A}$ was used to provide the external mechanical force. $\mathrm{c})$ A dilute $\mathrm{HCl}$ treated $\mathrm{WO}_{3}$ powder $(\sim 80 \mathrm{~nm})$ galinstan droplet was cut using a scalpel blade to realize two separate galinstan coated droplets. d) Sigmacote treated $\mathrm{Al}_{2} \mathrm{O}_{3}$ powder $(\sim 9 \mu \mathrm{m})$ coated galinstan droplet floating on water.

To test whether a liquid metal marble coated with hydrophobic particles can be suspended on the surface of water due to surface tension, we rolled a galinstan droplet on a powder bed of $\mathrm{Al}_{2} \mathrm{O}_{3}$ powder treated with Sigmacote to render it hydrophobic. We then placed the droplet on the surface of a beaker of water. Figure 3d shows this coated droplet suspended on the surface of water. Significant displacement of the water is evident due to the considerable weight of the liquid metal marble.

To test the behavior of liquid metal marbles under impact after free fall, we compared the behavior of an uncoated droplet of galinstan to a liquid metal marble (galinstan rolled on $80 \mathrm{~nm} \mathrm{WO}_{3}$ powder bed) after release from a height of $25 \mathrm{~mm}$ and impacting on a silicon surface. A high speed camera (1600 images per second) is used to record the image of the droplets during the fall and impact.

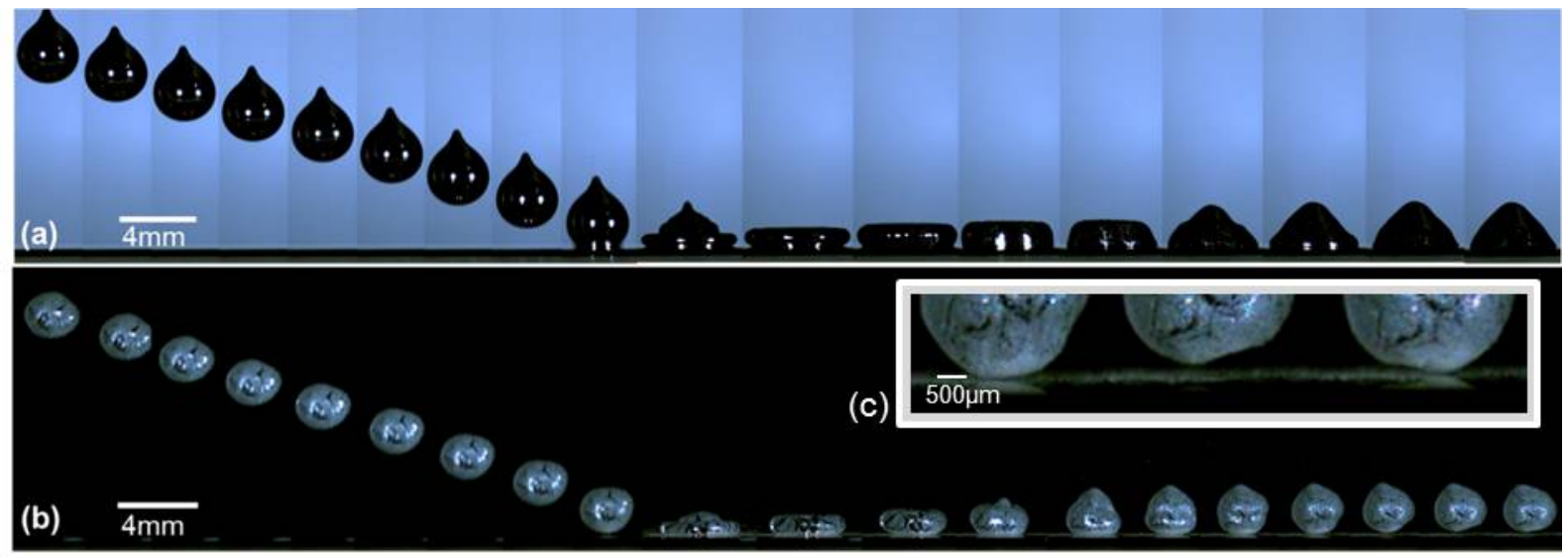


Submitted to

Figure 4: Successive snapshots of a) pure galinstan pendant drop and b) $\mathrm{WO}_{3}$ coated galinstan liquid marble dropped from a height of $25 \mathrm{~mm}$ on a silicon wafer surface, the time interval between two successive frames is $1.875 \mathrm{~ms}$ ) zoomed in photographs of the last three frames showing the bouncing of the galinstan liquid marble.

Figure 4a presents sequential images of the uncoated galinstan after being dispensed from a pipette, falling and impacting a Si surface. The droplet maintains a teardrop shape throughout the free fall which can be explained due to the oxide skin that is naturally formed on the surface of the liquid metal droplet in ambient conditions. At the impact, a crown-like rim having an obtuse contact angle is observed transforming into one with its angle of contact receding to an acute angle and is quickly transformed from a pendant shape to a more even conical shape. No rebound from the surface is observed. The free fall and impact video can been seen in the Supplementary Video SV1.

Figure 4b shows the coated galinstan liquid marble fall, after being released from a spatula, and impact on the same surface. The marble retains its almost spherical shape during its free fall. At the impact, a crown-like rim is again observed having an obtuse contact angle. Interestingly, we also observed significant tears on the coated surface together with the shedding of some of the particles at impact (clearly seen in the Supplementary Video SV2). The tears appear to close up and the marble is observed to regain its almost spherical shape as it rebounds from the surface. This is a characteristic that is not seen to the same extent in conventional aqueous liquid marbles. Figure $4 \mathbf{c}$ is the zoomed in photographs of the last three frames of Figure $4 \mathrm{~b}$ showing the rebound of the galinstan marble from the surface and its return. This characteristic can be more clearly seen in the Supplementary Video SV2. Upon return to the surface the marble rolls freely on the surface and behaves like a true liquid marble. 


\section{Submitted to}

\section{Electronic Properties}

An important property of the liquid metal droplets is the high electrical conductivity of the liquid metal itself. The use of uncoated liquid metal for so called 'soft electronics' has been reported previously, ${ }^{[12,13]}$ however, the corrosive nature of the liquid metal in contact with other metals imposes certain limitations. Our finding that liquid metals can be encapsulated within semiconducting and insulating coatings presents new opportunities for the realisation of soft electronic circuits without the need to directly contact the liquid metal itself. Sopecially, the semiconducting materials offer the possibility of establising liquid metalsemiconducting junctions that can be used in the formation of novel electronic devices.

In liquid metal marble contacts, in addition to the properties of the semiconducting powder, which covers most of the surface of the liquid metal, the native oxide layer that is naturally formed on the surface and sorrounding the rest of the liquid metal marble also play an important role in the properties of the junction. In order to understand the beheaviour of different types of pwoders and the influence of the native metal oxide layer, eight different experiments were conducted in order to invesigate the electronic properties of liquid metal marbles. In these experiments, we investigated the effect of the native oxide layer and the characteristics of liquid marbles coated with n-type $\left(\mathrm{WO}_{3}\right)$ and p-type $(\mathrm{CuO})$ semiconducting nanopowders in marble-metal and marble-marble configurations. Many other semiconducting poweder materials were also experimented but have not shown for berevity.

Firstly, to demonstrate that it is possible to form an electrical interface across the n-type semiconducting coating $\left(\mathrm{WO}_{3}\right)$ without the native oxide layer on the surface of the liquid metal, we conduct the experiment in a nitrogen filled glove box at room temperature. We treated the liquid metal droplet in diluted $\mathrm{HCl}$ within the glove box to ensure the droplet is native oxide layer free and then form the $\mathrm{WO}_{3}$ coated liquid metal marble (rolled on $80 \mathrm{~nm}$ 
Submitted to

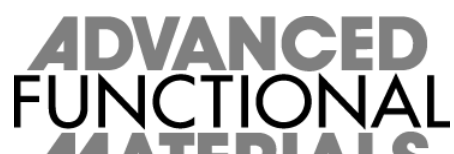

$\mathrm{WO}_{3}$ powder bed). The marble is then placed onto a gold coated silicon wafer. We then inserted a probe electrode into the liquid marble and measured the current-voltage $(I-V)$ characteristics of the electrical interface established from within the marble, across the particle coating and to the gold substrate. Four consecutive scans were performed measuring the current as the voltage was scanned from $-9 \mathrm{~V}$ to $+9 \mathrm{~V}$. Figure 5a presents a photograph of the marble-metal configuration and also the measured $I-V$ characteristics. The $I-V$ characteristics are observed to be similar with consecutive voltage sweeps and there is hardly any observable difference between sweeps.

The $I-V$ characteristics were obtained on the same marble after being exposed to ambient air (including 21\% oxygen) condiction and is presented in Figure 5b. In this case, it can be observed that the current obtained for large and positive voltages slightly increased initiall but then decreased after each voltage sweep.

The next pair of experiments were conducted with two $\mathrm{WO}_{3}$ coated marbles in contact resting on an insulating (Teflon) substrate (Figures 5c and 5d). In this pair, the first experiment was conducted with the galinstan droplets treated with dilute $\mathrm{HCl}$ before coating with $\mathrm{WO}_{3}$ nanopowder in a nitrogen filled glove box. Electrodes were inserted into the liquid metal marbles and the $I-V$ characteristics were recorded. Consecutive sweeps resulted in very similar responses as seen in Figure 5c. The consecutive experiment was conducted on the same marbles after being exposed to ambient air condition and the $I-V$ characteristics are presented in Figure 5d. In this case, however, the absolute current value obtained for both large negative and large positive voltages first increased but then significantly decreased with sequential voltage sweeps. 
Submitted to
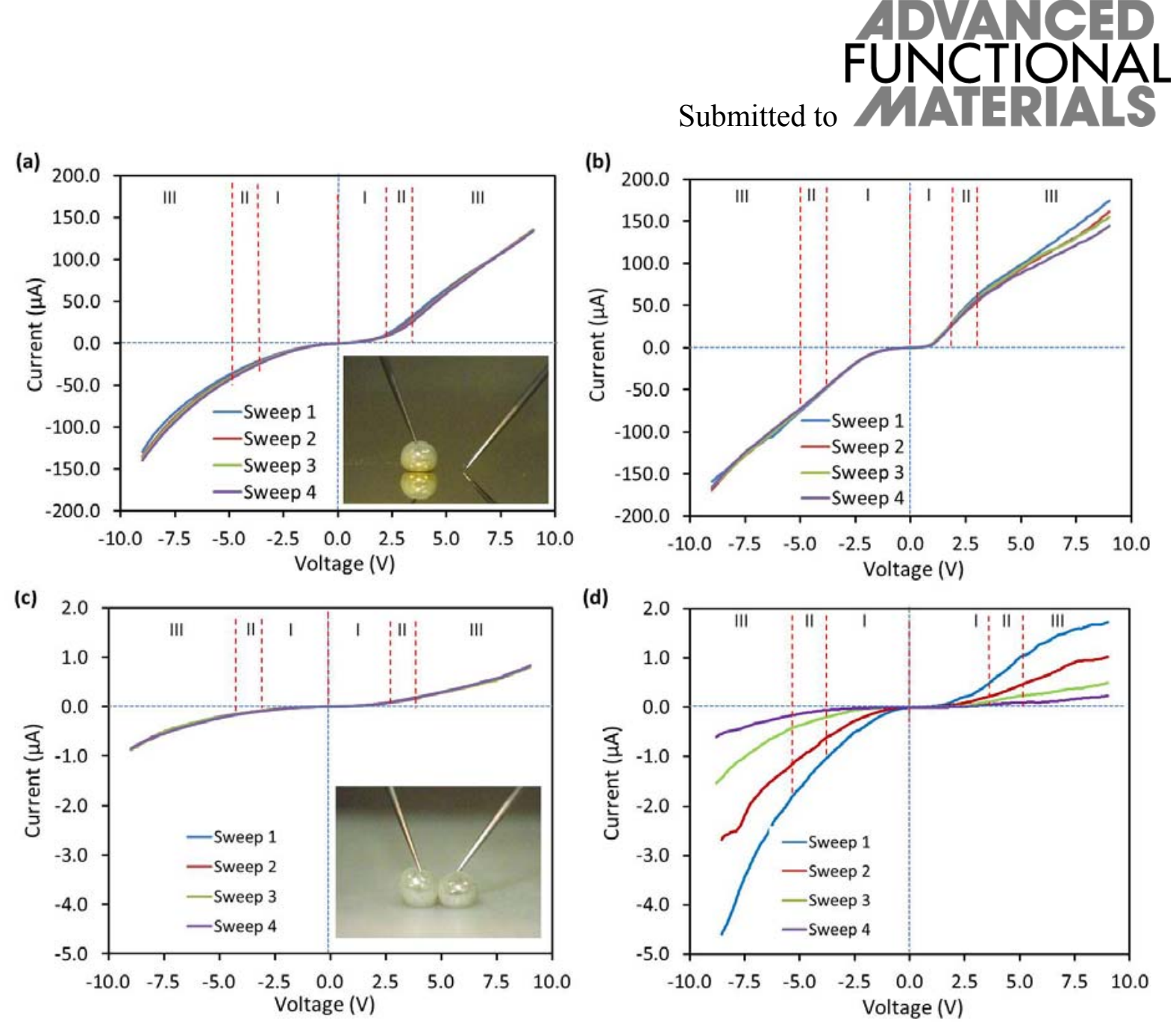

Figure 5: $I-V$ characteristics of the $\mathrm{WO}_{3}$ coated galinstan liquid marble - in contact with a gold substrate: a) in a nitrogen filled glove box to prevent oxidation, b) in ambient air condition; and in contact with another $\mathrm{WO}_{3}$ coated galinstan liquid marble: c) in a nitrogen filled glove box, $\boldsymbol{d})$ in ambient air condition. It is important to note that the same marbles were used for the experiment in the nitrogen filled glove box and in the ambient air condition measurements to allow the direct comparison of the I-V curves. The four consecutive voltage sweeps are labelled as sweeps 1,2,3 and 4 in the figures.

The $I-V$ characteristics behaviors in all cases of Figure 5 can be explained using wellestablished metal-semiconductor-metal (MSM) junction models. ${ }^{[28]}$ It is suggested that the current transport behavior of MSM junctions can be described using the thermo-ionic emission theory. The current in such a junction is due to a combination of electron and hole 
Submitted to
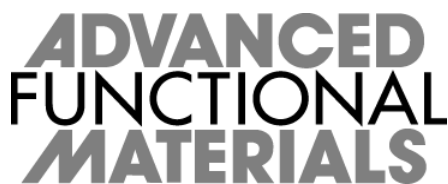

carriers. In Figures 5, we observe that the curves are very much similar to that of a solid state MSM curve which is defined for an intermediate $\mathrm{n}$-type semiconductor layer. The $I-V$ curves can be divided into "three" separate stages, labelled in Figure 5 as I, II and III:

In "Stage I", for small voltages $(V)$, when the depletion regions of two metal-semiconductor (MS and SM) junctions do not coincide, which is called the reach-through voltage $\left(V_{R T}\right)$, the main current density generated by the electron current is given by:

$$
J_{n} \approx J_{n s} e^{\beta \Delta \phi_{n 1}}\left(1-e^{-\beta V}\right), \quad V<V_{R T} .
$$

Most of the voltage drops on the first junction which is reverse biased and is due to the saturation current $\left(J_{n s}\right)$ and $\beta=q / k T$, in which $k$ is the Boltzmann's constant, $q$ is the charge of an electron and $T$ is the temperature. $\Delta \Phi_{n 1}$ is the change in the barrier height, which is obtained using:

$$
\Delta \phi_{n 1}=\sqrt{\frac{q E}{4 \pi \varepsilon_{s}}}
$$

in which:

$$
E=\sqrt{\frac{2 q N_{D}}{\varepsilon_{s}}\left(V+V_{D}\right)}
$$

where $\varepsilon_{S}$ is the permittivity of the medium, $N_{D}$ is the concentration of electrons per volume and $V_{D}$ is the built in voltage.

In "Stage II", when the applied voltage is larger than the reach-through voltage and smaller than when the band becomes flat $\left(V_{F B}\right)$, the current density results from the combination of electron and hole carriers:

$$
J \approx J_{n s} e^{\beta \Delta \phi_{n 1}}+J_{p s}\left[e^{-\frac{\beta\left(V-V_{F B}\right)^{2}}{4\left(V_{F B}\right)}}\right], \quad V_{R T}<V<V_{F B}
$$

in which $J_{p s}$ is the saturation current for holes. 
Submitted to

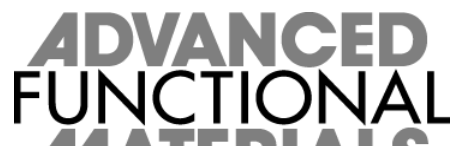

IATERAS

In "Stage III", when the applied voltages is greater than the flat-band voltage, the current density mostly results from the hole (minority) current:

$$
J_{p} \approx J_{p s} e^{\beta \Delta \phi_{p 2}}, \quad V>V_{F B}
$$

in which $\Delta \Phi_{p 2}$ is the change in the barrier height for the second junction.

The $I-V$ curves demostrated in Figure 5 can all be apprximated by equations (1) to (5) using different oxide layer thicknesses. As such, we acsibe the change in the I-V curve after the exposure to ambient air and sequential voltage sweeps to the changes of the oxide layer thickness as follows.

It can be observed that the $I-V$ characteristics remained unchanged in the absence of the native oxide skin (in nitrogen environment) for both Figure 5a and 5c. It was expected to see a decrease of current after the exposure to the ambient air due to the formation of the native oxide. However, the opposite was obsreverd. By comparing the $I-V$ characteristics obtained in Figure $5 \mathrm{a}$ and the first sweep of and $5 \mathrm{~b}$, we observed an increase in the maximum current in the experiment conducted in ambient conditions. The nano pawder does not cover the whole surface of the metal droplet so there exist an air-gap. In addition, the nanopowders sharp edges and projection also enhance the formation of plasma by enhancing the local electric field. ${ }^{[2]}$ As a result, we suggest that this increase in current is due ionisation of the gas species in the air-gap beween the metal contact and the liquid metal that generates an extra path for the passage of current. However, the existance of the oxygen ions generated by the plasma increase the thcikness of the native oxide in time. As a resut, the current decreases in the consecutive sweeps (Figure 5b). A similar observation can be made in Figure 5c and 5d. However, the decrease in current after the suncequent sweeps is much more prominenst. We acsibe this to the existance of the enhanced electric field by both sides of the contact that increase the rate of the oxidation and hence thicker native oxide in the process. 
Submitted to

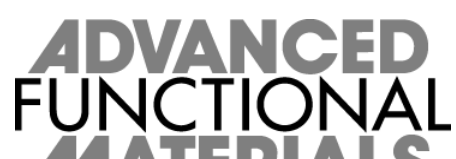

There are significant differences between Figures 5a and 5c: Firstly, Stage II is shifted to the right side in the $I-V$ curves for the two marbles (Figure 5b) which is caused by the prevalence of the second term in Equation (4) at lower voltages. Secondly, the current in the two marble case of Figure $5 \mathrm{~b}$ is much smaller than for the single marble case of Figure $5 \mathrm{a}$ due to the increased thickness of the intermediate semiconducting layer.

Nextly, we repeat the $I-V$ characteristic measurements on a p-type semiconducting powder $(\mathrm{CuO})$ using the same experimental procedure, described to obtain the characteristics in Figure 5. Figures $6 \mathbf{a}$ and $\mathbf{6 b}$ presents the characteristics of a liquid metal marble configuration in the absence of the native oxide layer and in the presence of the oxide layer, respectively. Figures 6c and $\mathbf{6 d}$ present the characteristics of a marble-marble configuration in the absence of the native oxide layer and in the presence of the oxide layer respectively.

Similar to the case of the n-type materal $\left(\mathrm{WO}_{3}\right)$ the p-type material $(\mathrm{CuO})$ also follows equations (1) to (5) and calsulations show that there is no significant changes in the behaviour of the $I-V$ curves execpt the inset of "Stages". However, as the electrical conductivity of $\mathrm{CuO}$ particles is smaller than that of $\mathrm{WO}_{3}$ partices the saturation current is significantly smaller, which result is an overall smaller current. 
(a)

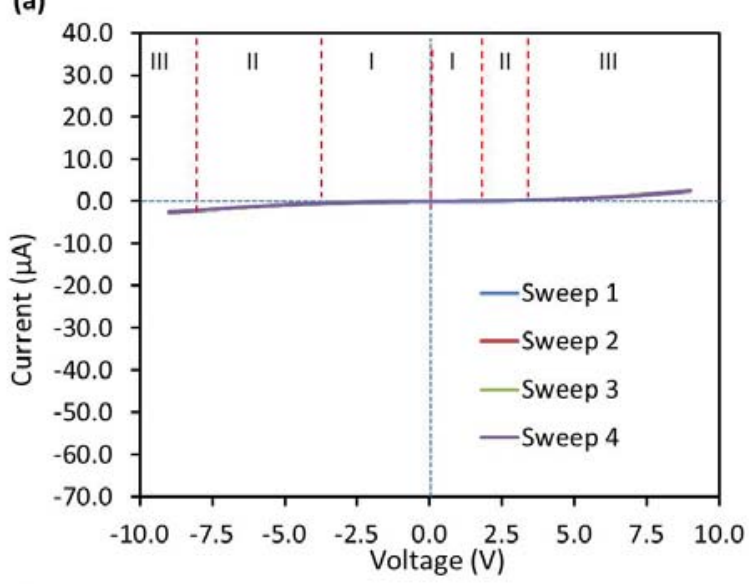

(c)

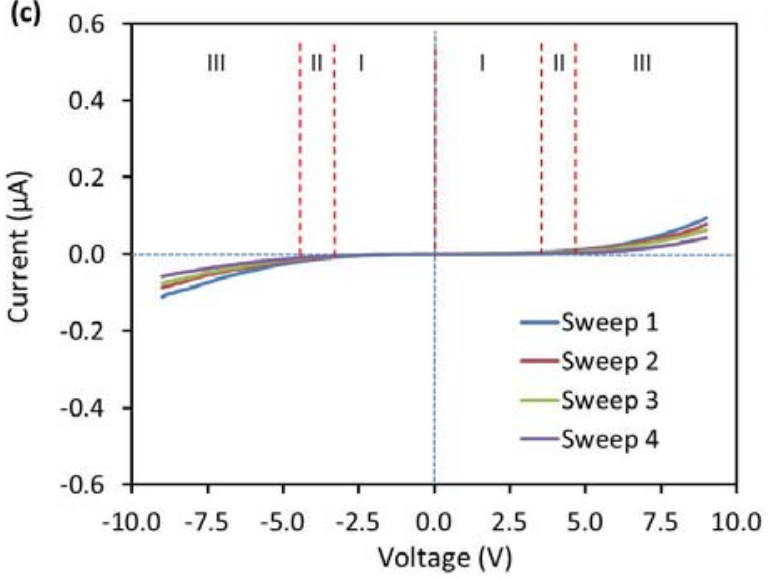

(b)

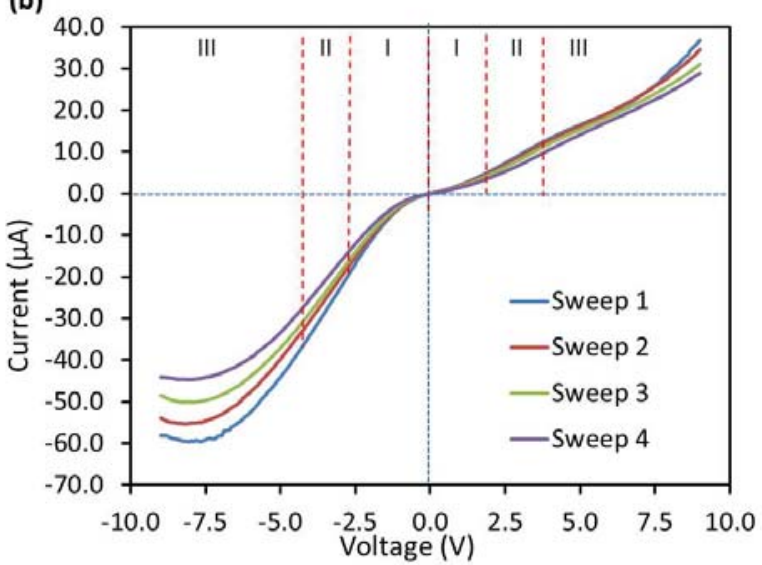

(d)

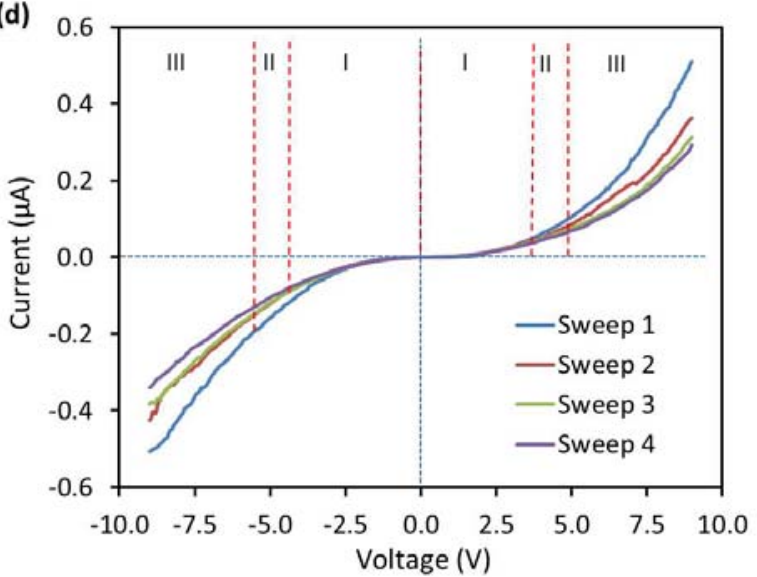

Figure 6: I-V characteristics of the CuO coated galinstan liquid marble - in contact with a gold substrate: a) in a nitrogen filled glove box to prevent the formation of a native oxide layer, $\boldsymbol{b})$ in ambient air condition; and in contact with another $\mathrm{CuO}$ coated galinstan liquid marble $\boldsymbol{c}$ ) in a nitrogen filled glove box to prevent the formation of a native oxide layer, $\boldsymbol{d}$ ) in ambient air condition. The four consecutive voltage sweeps are labelled as sweep 1,2,3 and 4.

These preliminary results indicate that it is possible to form simple circuits using these liquid metal marbles and crudely covered semiconducting nano-particles coatings. It is possible to form many other types of junctions including metal/p-type semiconductor/n-type semisocnsuctor/ metal junctions with interesting $I-V$ characteristics. However, this is beyond the scope of this paper. 


\section{Submitted to}

\section{Electrochemical Properties}

As a final demonstration of the utility of these liquid metal marbles, we investigate their use for heavy metal ion sensing. Galinstan has been used as a heavy metal ion sensing material and has been shown as an attractive alternative to mercury, possessing a high hydrogen overpotential, good electrochemical behavior, simple surface renewal at drop-formation, which allows high reproducibility, and the non-toxicity of this alloy. ${ }^{[24]}$ Here, we show that it is possible to dramatically enhance the sensitivity by coating the liquid metal with semiconducting nanoparticles.

We used a Hanging Galinstan Drop Electrode (HGDE) configuration similar to that previously reported, ${ }^{[20]}$ with the difference being that the liquid metal is coated by $\mathrm{WO}_{3}$ nanoparticles. In this investigation we used emersion in a colloidal suspension of $80 \mathrm{~nm} \mathrm{WO}_{3}$ nanoparticles to form the coating. Four different cases were studied, uncoated galinstan and galinstan immersed in suspensions of $0.1 \mathrm{~g}, 0.4 \mathrm{~g}$ and $0.7 \mathrm{~g}$ of $\mathrm{WO}_{3}$ per $50 \mathrm{ml}$ of DI water. Each case should result in an increased amount of $\mathrm{WO}_{3}$ being dispersed over the surface of the galinstan marble. A schematic of the experimental setup is shown in Figure 6a. The HGDE and a reference electrode were immersed in an electrolyte containing both $\mathrm{Pb}$ and $\mathrm{Cd}$ ions. $\mathrm{A}$ voltage was applied between them and the current was measured.

Figure $6 \mathbf{b}$ presents the differential pulse voltammogram of $\mathrm{Pb}^{2+}$ and $\mathrm{Cd}^{2+}$ in an acetic buffer solution. We observe selectivity for $\mathrm{Pb}$ and $\mathrm{Cd}$ ions that are present in the solution. We also observe an enhancement in the sensitivity for detection the metal ions on the coated marbles when compared to that of the uncoated galinstan drop. The particle density on the surface influences the enhancement. Moreover, the current peaks are observed to be shifted to a lower potential in the case of coated liquid metal electrodes. This is a desirable effect since it expands the negative potential window for heavy metal ion sensing. 


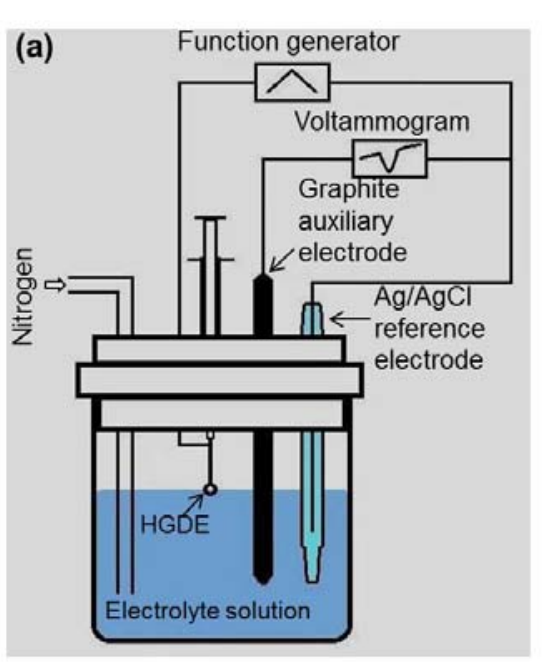

Submitted to

FUNCTIONAL

Voltage (V)

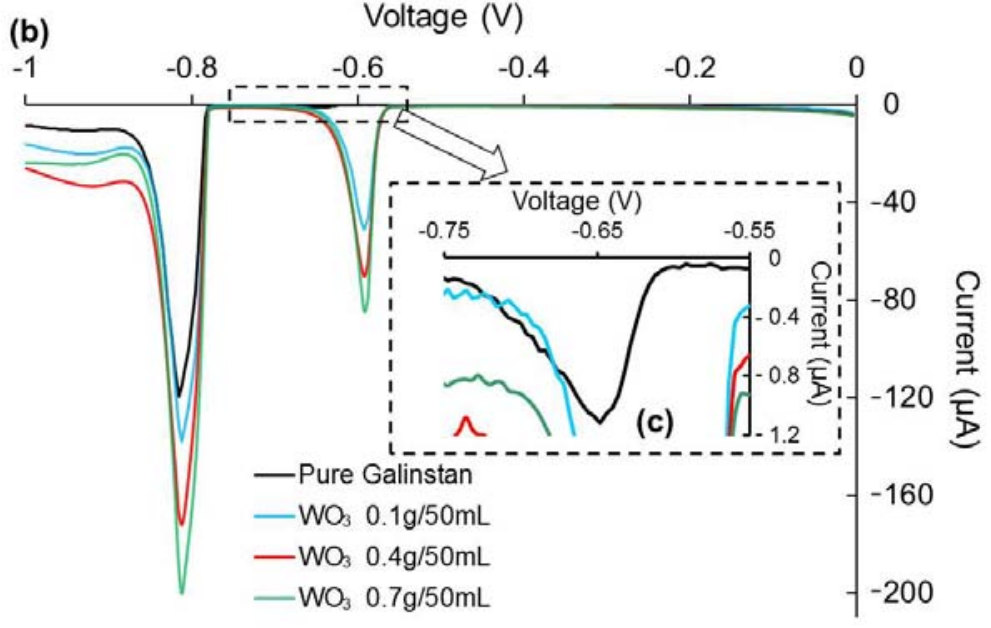

Figure 6: a) Schematic of the experimental setup using Hanging Galinstan Drop Electrode (HGDE) for heavy metal ion sensing application. b) Differential pulse voltammogram (DPV) of $\mathrm{Pb}^{2+}(10 \mathrm{mmol} / \mathrm{L})$ and $\mathrm{Cd}^{2+}(10 \mathrm{mmol} / \mathrm{L})$, obtained with $\mathrm{HGDE}$ coated with $\mathrm{WO}_{3}$ nanopowder using the submerging technique with the nanoparticles dispersed in DI water having concentrations of $0.1 \mathrm{~g}$ in $50 \mathrm{ml}, 0.4 \mathrm{~g}$ in $50 \mathrm{ml}$ and $0.7 \mathrm{~g}$ in $50 \mathrm{ml}$ at $\mathrm{pH}=6.0$. c) A zoom-in of the response of the DPV sensing $\mathrm{Pb}^{2+}$ ions using pure galinstan in the HGDE.

It is expected at $\mathrm{pH} 6.0$ and the voltage range employed here that the electrochemical reduction of the native oxide layer that is normally formed in ambient air condition on the surface of this liquid metal occurs. ${ }^{[30]}$ There is also a significant shift in the onset potential to less negative values and increase in current magnitude for the reduction of $\mathrm{Pb}$ ions onto the galinstan surface when coated with $\mathrm{WO}_{3}$ particles. The response is also sharper when compared to the unmodified surface. The possibility of a change in the surface area of galinstan or the diffusion of $\mathrm{Pb}^{2+}$ ions to the electrode surface accounting for this was investigated by coating the galinstan drop with non-conductive particles such as Teflon particles where no significant enhancement in current or change in onset potential were observed (Supplementary S4). This indicates that there is a large reduction in the 
Submitted to

overpotential required for the reduction of $\mathrm{Pb}^{2+}$ to $\mathrm{Pb}^{0}$ on the $\mathrm{WO}_{3}$ modified surface and possibly more facile amalgamation of $\mathrm{Pb}^{0}$ with galinstan. The creation of a triple phase galinstan $/ \mathrm{WO}_{3}$ /electrolyte region may be crucial in creating hot spots, where the transfer of electrons is facilitated as observed in many nanostructured electrode studies. Indeed, the magnitude of the response increases with an increased loading of $\mathrm{WO}_{3}$ on the galinstan surface.

When the surface coverage of $\mathrm{WO}_{3}$ is significantly increased (multilayer $\mathrm{WO}_{3}$ particles coating on the liquid metal marble presented in the Supplementary S5), there is a large increase in the reduction current and a new process at ca. $-0.40 \mathrm{~V}$, which is significantly reduced during subsequent voltage sweeps. The latter may be attributed to the intercalation of electrolyte cations into $\mathrm{WO}_{3}$ as indicated by the change in colour of the sample in this potential range (Supplementary S6). This process was also observed to occur in the absence of $\mathrm{Pb}^{2+}$ ions. This is followed by a large increase in cathodic current at $-0.90 \mathrm{~V}$ until the end of the sweep which is due to the hydrogen evolution reaction at $\mathrm{WO}_{3}$.

From a practical viewpoint, this composite shows promise in that not only the sensitivity for heavy metal detection is increased as observed for $\mathrm{Pb}^{2+}$ detection but it also demonstrates selectivity as $\mathrm{Cd}^{2+}$ ions were detected at a distinctly more negative potential of ca. $-0.80 \mathrm{~V}$. Interestingly in the latter case there is no shift in the onset potential for the reduction of $\mathrm{Cd}^{2+}$ to $\mathrm{Cd}^{0}$ at the modified galinstan electrode but there is a significant increase in the current magnitude. This indicates that the electron transfer process for the reduction of $\mathrm{Cd}^{2+}$ ions to $\mathrm{Cd}^{0}$ or amalgamation of $\mathrm{Cd}$ with galinstan is not influenced to the same extent as observed in the case of $\mathrm{Pb}$. However the presence of the initially formed $\mathrm{Pb}$ in the galinstan may be accountable for this observation. These measurements were repeatable on different similar size and coated marbles (Supplementary S7) 


\section{Conclusions and discussions}

Submitted to

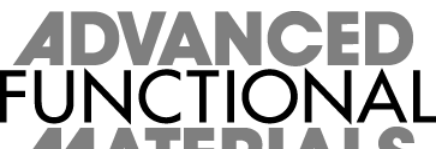

MATERIALS

We have introduced 'liquid metal marbles' formed from droplets of the liquid metal galinstan coated with insulators and semiconductors. We have demonstrated some of the unique physical, electronic and electrochemical properties of these liquid metal marbles. We realized that physically there are similarities and differences between conventional liquid marbles and liquid metal marbles. High surface tension, the formation of a native oxide and high density of liquid metal marbles are the properties that give them their extraordinary physical features. Electronic properties of liquid metal marbles were characterized to observe interesting MSM junction behavior which can be extended into many applications in soft electronic devices. It was also shown that the nanoparticle coated liquid metal marbles can provide enhanced sensing of heavy metals, presenting a new avenue for investigation into safe and highly sensitive systems for the detection of low concentrations of heavy metal ions with high selectivity.

There are many other possible applications that can be considered. For instance, the coatings provide a non-stick property to the liquid metal marbles and hence can be used as conductive lubricants. They should operate much better than the conventional liquid marbles in tolerating heavy load and pressure as their surface tension is much higher. The high evaporation point and low vapor pressure of liquid metal marbles can be some of the unique properties allowing them to operate at very high temperatures as well as in a vacuum. Conventional liquid marbles can be made conductive, but only through use of ionic liquids which are very poor subsitutes for traditional electrodes. Conversely, liquid metal marbles have conductivities approaching solid metals and can be coated with a wide variety of both insulating and of semiconducting materials enabling many different circuit elements, including diodes and transistors, that could be considered for soft electronic circuits. These preliminary 
Submitted to

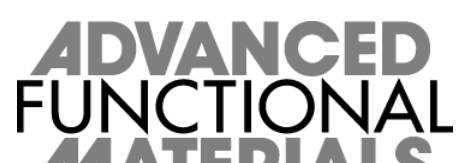

demonstrations certainly illustrate promise of what this new approach to the use of liquid metals can offer for future scientific research and technological applications.

\section{Experimental Section:}

Realisation of liquid metal marbles:

Galinstan (Geratherm Medical AG, Geschwenda, Germany) was used to realise the liquid metal marble. Two different approaches are presented in this work.

Method 1: A droplet of galinstan was dispensed through a syringe on a powder bed and rolled until the entire surface of the droplet was covered by the powder material. Different powder materials including $\mathrm{WO}_{3}, \mathrm{In}_{2} \mathrm{O}_{3}, \mathrm{ZnO}, \mathrm{Al}_{2} \mathrm{O}_{3}$ and $\mathrm{TiO}_{2}$ (China Rare Ltd.), Teflon (Sigma Adrich) and carbon nanotubes (CheapTubes Ltd.) were used.

Method 2: A colloidal solution of nano size powder was formed by dispensing a specific quantity of a powder material in $50 \mathrm{ml}$ of de-ionised (DI) water and dispersing it using a high intensity ultrasonic processor (GEX500) for $30 \mathrm{~min}$. A droplet of galinstan is then submerged into the colloidal solution for $5 \mathrm{~s}$.

\section{Characterization:}

SEM images were taken using a FEI Nova NanoSEM. The contact angles were measured using a Dataphysics OCA20 contact angle measuring system at ambient temperature. High speed imaging of the free fall and impact images and films were taken using a high-speed video camera (Phantom v4.3, Vision Research Inc.) fitted with a Navitar 12mm Zoom 6000 lens, $0.67 \times$ adapter, and $0.25 \times$ lens attachment. Events were captured at 1600 frames per second, with a resolution of $608 \times 600$ pixels and an exposure time of $248 \mu$ s for each frame.

\section{Electronic Properties:}

For investigating the electrical characteristics of liquid metal marbles, the $I-V$ characteristic curves were obtained by using a Lab-view controlled source meter (Keithley 2406, Keithley Instruments Inc.). Tungsten probes attached to micromanipulators were employed to connect 
Submitted to

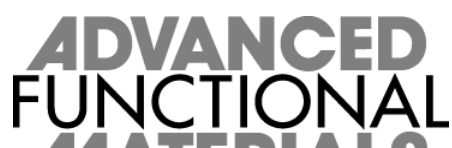

14 A ERALS

with the liquid metal marbles and the gold coated substrates for the different electrical measurements.

\section{Electrochemical Properties:}

For heavy metal ion sensing, all measurements were performed using a $\mathrm{CH}$ Instruments (CHI 413A) electrochemical analyser. The reference electrode was $\mathrm{Ag} / \mathrm{AgCl}$ (aqueous $3 \mathrm{M} \mathrm{KCl}$ ) and an inert graphite rod ( $3 \mathrm{~mm}$ diameter, Johnson Matthey Ultra ' $F$ ' purity grade) was used as the auxiliary electrode, based on the three-electrode configuration for the electrochemical measurements. All chemicals were of analytical grade purity and all aqueous solutions were prepared using Milli-Q water. All electrochemical measurements commenced after degassing the electrolyte solutions with nitrogen for at least 10 min prior to any measurement. Acetate buffer solutions with a $\mathrm{pH}$ of 6.0 were used as the supporting electrolyte, which was prepared by dissolving a 33.33g sample of ammonium acetate (Ajax Finechem) in $100 \mathrm{~mL}$ of Milli-Q water. The $\mathrm{pH}$ was corrected by adding acetic acid (99.7\%, Ajax Finechem) and measured using a microprocessor controlled $\mathrm{pH}$ meter (PH 213, HANNA Instruments). Lead ions

$\left(\mathrm{Pb}^{2+}\right)$ and cadmium ions $\left(\mathrm{Cd}^{2+}\right)$ were incorporated in the supporting electrolyte by dissolving lead (II) acetate 3-hydrate (BDH, AnalaR) and cadmium (II) nitride 4-hydrate (Ajax Finechem). The methodology adopted for the electrochemical measurements was differential pulse polarography (DPP) with a pulse amplitude of $-50 \mathrm{mV}$ and a pulse period of $0.5 \mathrm{~s}$.

\section{References}

[1] P. Aussillous, D. Quere, Nature 2001, 411, 924.

[2] P. Aussillous, D. Quéré, Proceedings of the Royal Society A: Mathematical, Physical and Engineering Sciences 2006, 462, 973.

[3] E. Bormashenko, Y. Bormashenko, A. Musin, Journal of Colloid and Interface

Science 2009, 333, 419. 
[4] E. Bormashenko, Y. Bormashenko, A. Musin, Z. Barkay, ChemPhysChem 2009, 10, 654.

[5] L. Gao, T. J. McCarthy, Langmuir 2007, 23, 10445.

[6] A. Venkateswara Rao, M. M. Kulkarni, S. D. Bhagat, Journal of Colloid and Interface Science 2005, 285, 413.

[7] T. Arbatan, L. Li, J. Tian, W. Shen, Advanced Healthcare Materials 2012, 1, 80.

[8] N. Eshtiaghi, K. P. Hapgood, Powder Technology 2012, 223, 65.

[9] T. Arbatan, W. Shen, Langmuir 2011, 27, 12923.

[10] E. Bormashenko, Current Opinion in Colloid \&amp; Interface Science 2011, 16, 266.

[11] P. Sen, K. Chang-Jin, Industrial Electronics, IEEE Transactions on 2009, 56, 1314.

[12] H.-J. Koo, J.-H. So, M. D. Dickey, O. D. Velev, Advanced Materials 2011, 23, 3559.

[13] J.-H. So, H.-J. Koo, M. D. Dickey, O. D. Velev, Advanced Functional Materials 2012, 22,625 .

[14] L. Tingyi, P. Sen, K. Chang-Jin, Microelectromechanical Systems, Journal of 2012, 21, 443.

[15] B. L. Mellor, N. A. Kellis, B. A. Mazzeo, Review of Scientific Instruments 2011, 82, 046110.

[16] S. Cheng, A. Rydberg, K. Hjort, Z. Wu, Applied Physics Letters 2009, 94, 144103.

[17] M. Kubo, X. Li, C. Kim, M. Hashimoto, B. J. Wiley, D. Ham, G. M. Whitesides, Advanced Materials 2010, 22, 2749.

[18] J.-H. So, J. Thelen, A. Qusba, G. J. Hayes, G. Lazzi, M. D. Dickey, Advanced Functional Materials 2009, 19, 3632.

[19] K. Hyun-Joong, T. Maleki, W. Pinghung, B. Ziaie, Microelectromechanical Systems, Journal of 2009, 18, 138.

[20] H.-J. Kim, C. Son, B. Ziaie, Applied Physics Letters 2008, 92, 011904. 
Submitted to

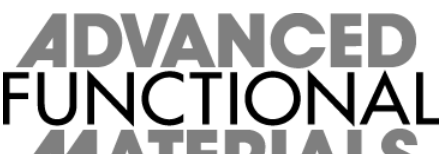

[21] A. C. Siegel, S. S. Shevkoplyas, D. B. Weibel, D. A. Bruzewicz, A. W. Martinez, G.

M. Whitesides, Angewandte Chemie International Edition 2006, 45, 6877.

[22] C. Chung-Hao, J. Whalen, D. Peroulis, "Non-Toxic Liquid-Metal 2-100 GHz MEMS

Switch", in Microwave Symposium, 2007. IEEE/MTT-S International, 3-8 June 2007, 2007.

[23] M. R. Khan, G. J. Hayes, J.-H. So, G. Lazzi, M. D. Dickey, Applied Physics Letters

2011, 99, 013501.

[24] P. Surmann, H. Zeyat, Analytical and Bioanalytical Chemistry 2005, 383, 1009.

[25] R. G. Burton, R. A. Burton, Components, Hybrids, and Manufacturing Technology, IEEE Transactions on 1988, 11, 112.

[26] L. Tingyi, P. Sen, K. Chang-Jin, "Characterization of liquid-metal Galinstan for droplet applications", in Micro Electro Mechanical Systems (MEMS), 2010 IEEE 23rd International Conference on, 24-28 Jan. 2010, 2010.

[27] V. Kocourek, C. Karcher, M. Conrath, D. Schulze, Physical Review E 2006, 74, 026303.

[28] S. M. Sze, D. J. Coleman Jr, A. Loya, Solid-State Electronics 1971, 14, 1209.

[29] J. Yu, M. Shafiei, W. Wlodarrski, Y. X. Li, K. Kalantar-zadeh, J. Phys. D: Appl. Phys. 2010, 43, 025103.

[30] J.-H. So, M. D. Dickey, Lab on a Chip 2011, 11, 905.

Received: ((will be filled in by the editorial staff))

Revised: ((will be filled in by the editorial staff)) Published online: ((will be filled in by the editorial staff)) 


\section{Supplementary Section}

Submitted to

\section{ADVANCED FUNCTIONAL \\ 14 1 1}

S1. SEM images of the surface of galinstan coated in different concentration of colloidal solution
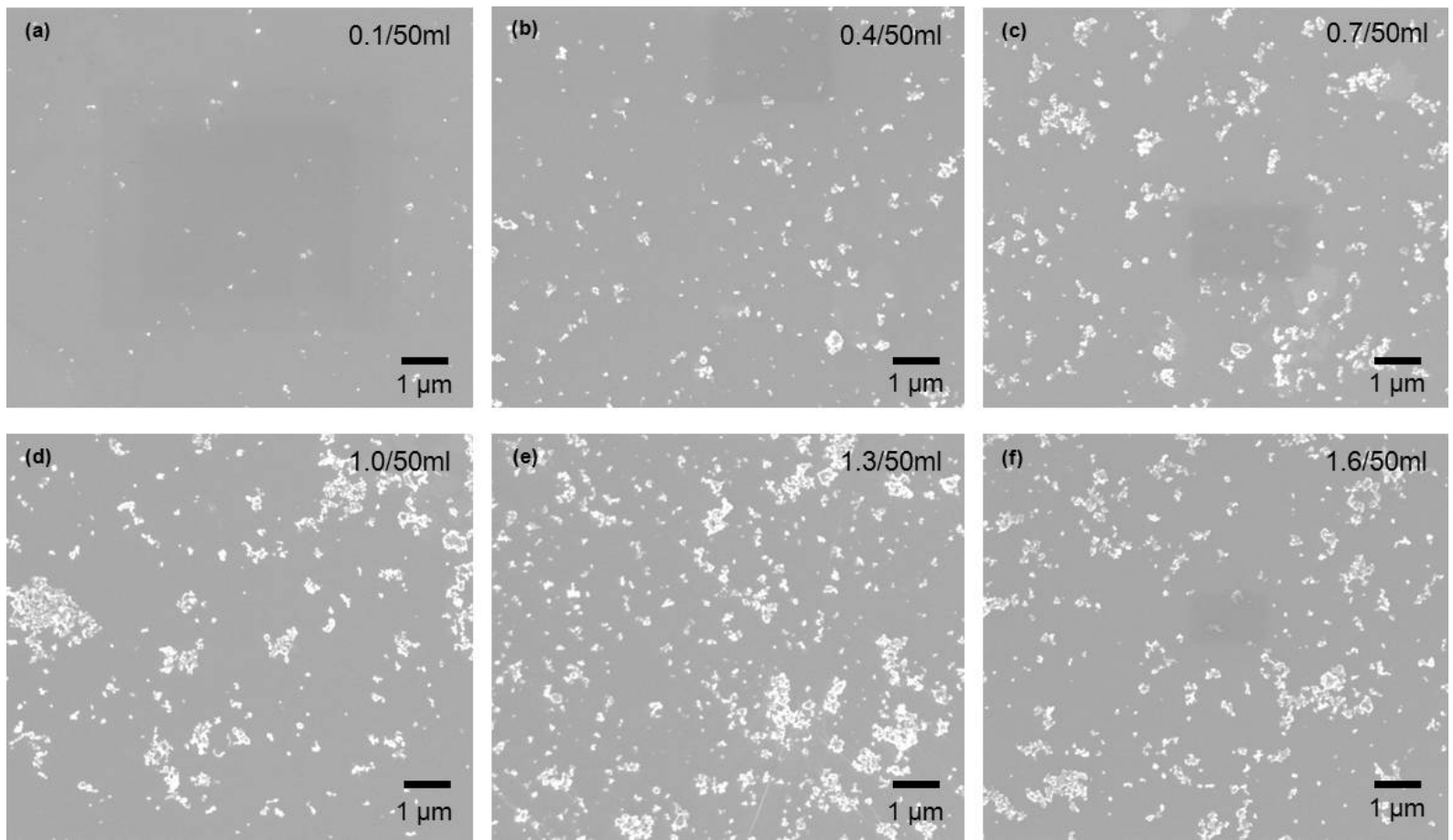

A droplet of galinstan coated in (a) $0.1 \mathrm{~g}$, (b) $0.4 \mathrm{~g}$, (c) $0.7 \mathrm{~g}$, (d) $1.0 \mathrm{~g}$, (e) $1.3 \mathrm{~g}$, and (f) $1.6 \mathrm{~g}$ of $\mathrm{WO}_{3}$ dispersed in $50 \mathrm{ml}$ of DI water.

S2. Statistical plot of the surface area to particle coverage ratio for 5 different concentrations of $\mathrm{WO}_{3}$ colloidal dispersions.

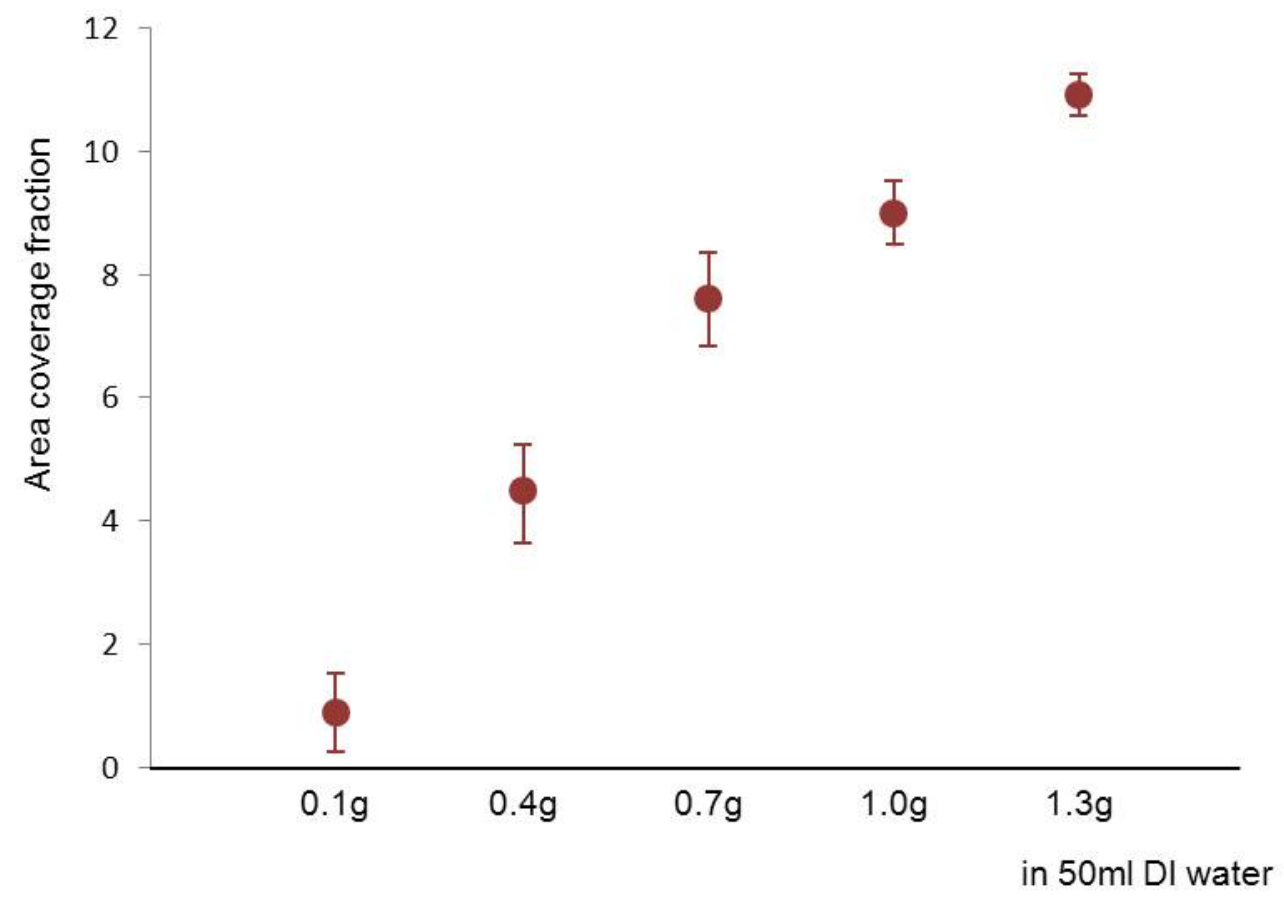




\section{Submitted to

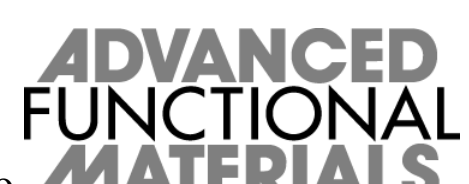

S3. (A) The sparsley coated marble is rolled on a normally wetting surface.

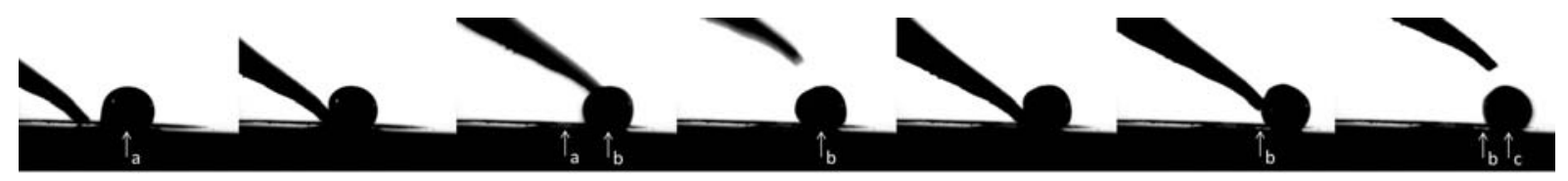

S3. (B) Galinstan has a contact angle greater than $>175^{\circ}$ on the bed of different type powder materials. The contact angle was measured using Dataphysics, the contact angle measurement system.

$\mathrm{Al}_{2} \mathrm{O}_{3}$

$$
\ln _{2} \mathrm{O}_{3}
$$
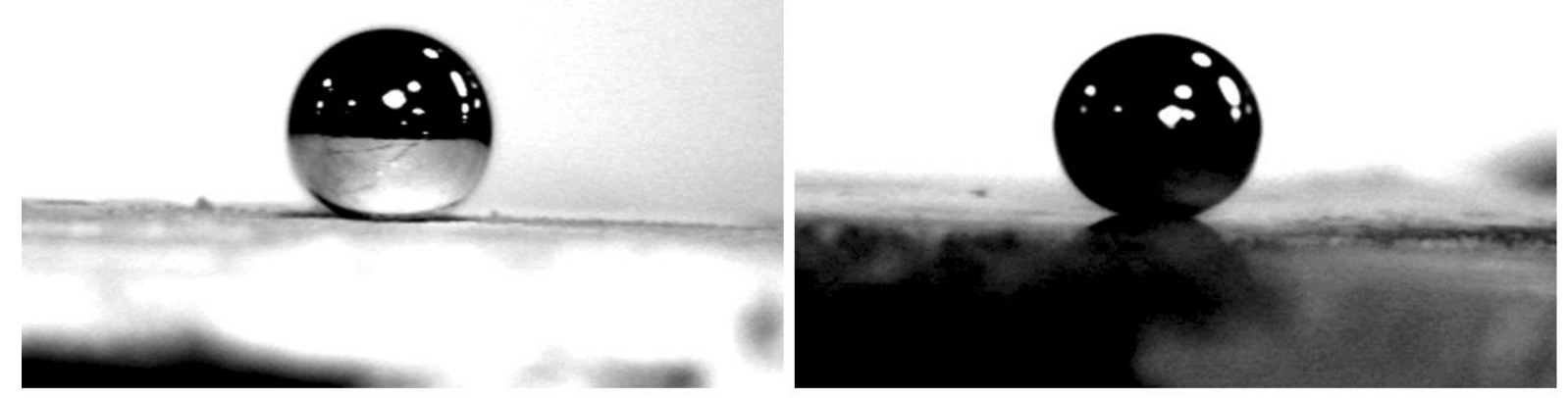

Teflon

$\mathrm{WO}_{3}$
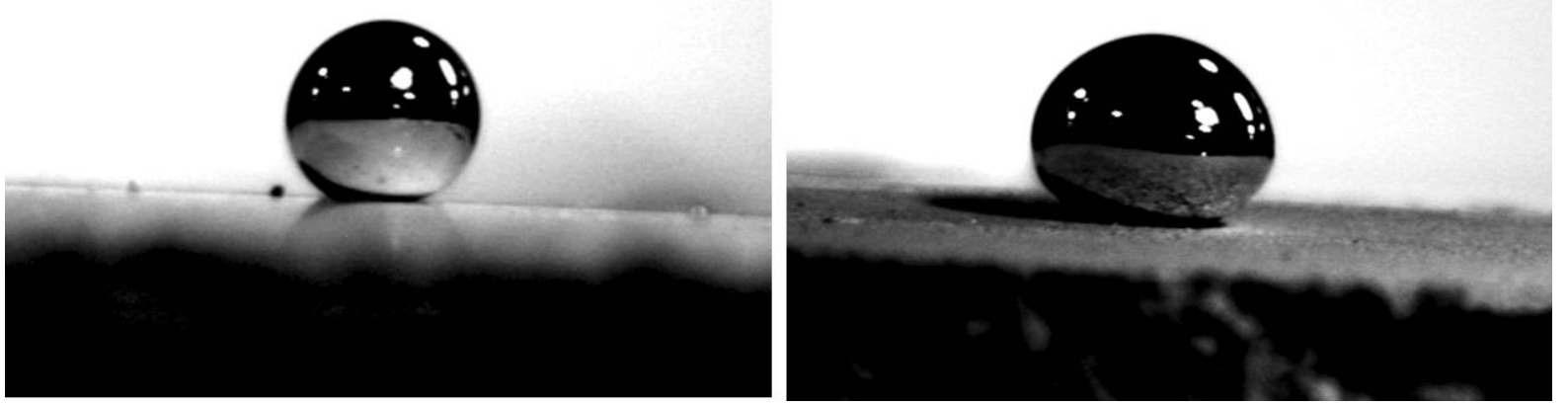

S4. Electrochemical heavy metal ion sensing with Teflon coated galinstan droplet using HGDE. The differential pulse voltammogram of $\mathrm{Pb}^{2+}(10 \mathrm{mmol} / \mathrm{L})$ and $\mathrm{Cd}^{2+}(10 \mathrm{mmol} / \mathrm{L})$ in an acetic buffer solution ( $\mathrm{pH}=6.0)$ using $1 \mu \mathrm{m}$ size teflon coated liquid metal marbles. 


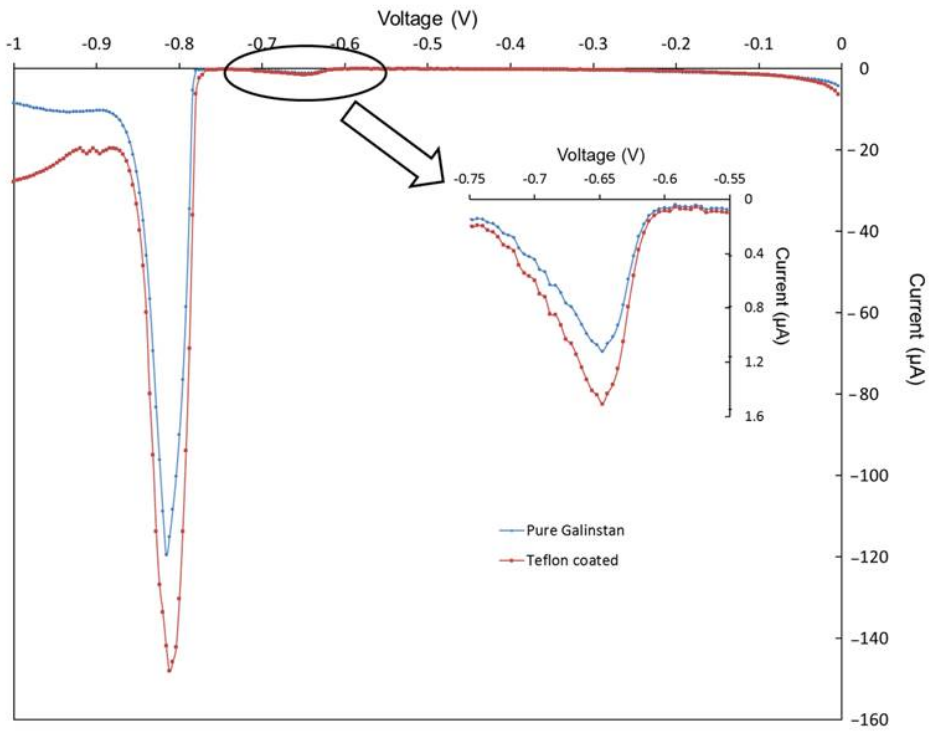

S5. Electrochemical heavy metal ion sensing with multilayer $\mathrm{WO}_{3}$ coated galinstan droplet using HGDE. The differential pulse voltammogram of $\mathrm{Pb}^{2+}(10 \mathrm{mmol} / \mathrm{L})$ and $\mathrm{Cd}^{2+}(10 \mathrm{mmol} / \mathrm{L})$ in an acetic buffer solution ( $p H=6.0)$. Results presented are three consecutive sweeps on the same droplet.

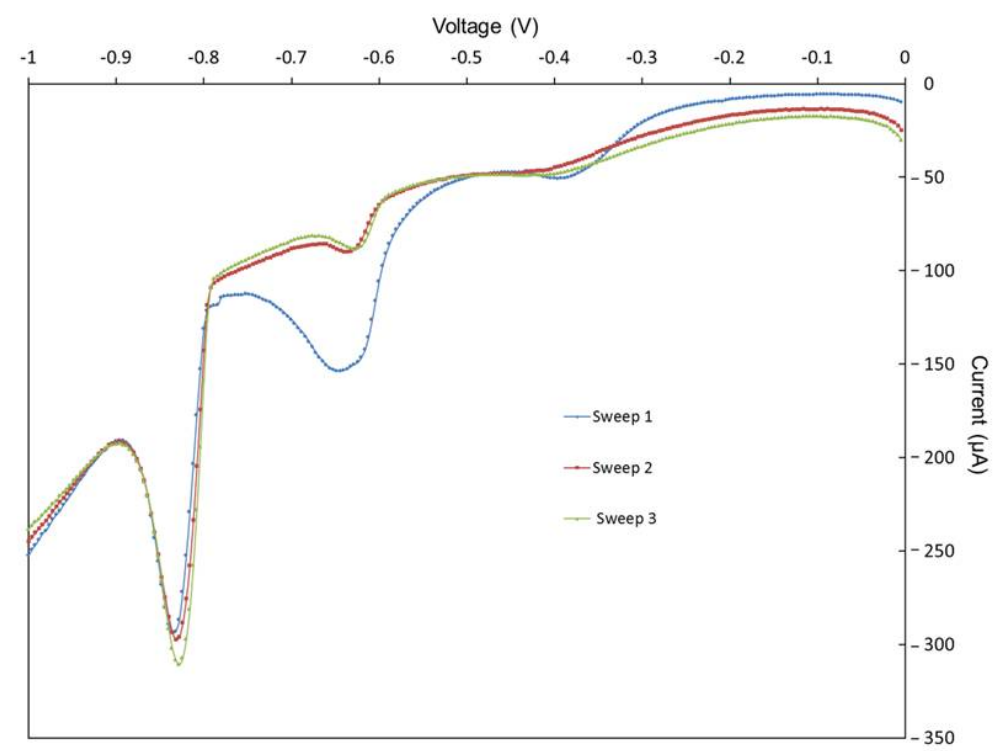

S6. Intercalation of electrolyte cations into $\mathrm{WO}_{3}$ indicated by the change in colour of the coating to Prussian blue (due to the intercalation process):

Multilayer $\mathrm{WO}_{3}$ coated hanging galinstan liquid marble electrode a) before electrochemical analysis and $\mathbf{b}$ ) after one voltage sweep. 

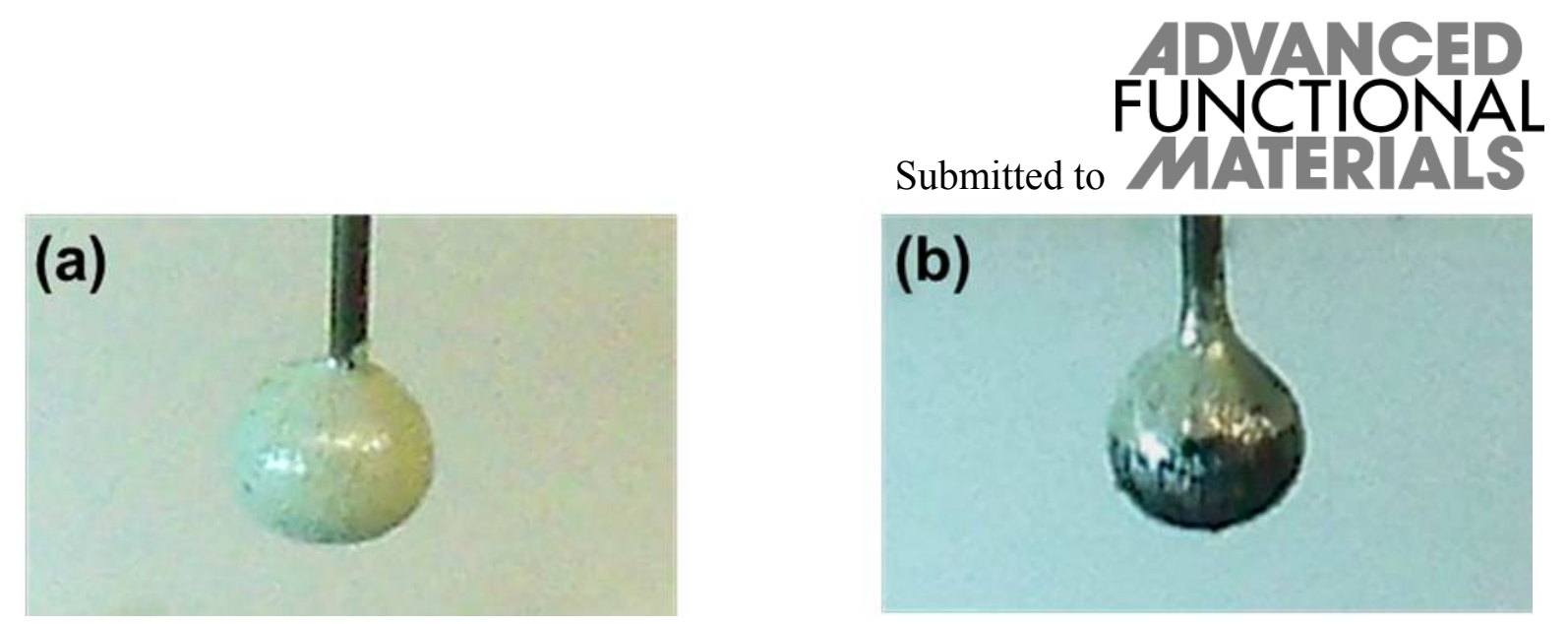

S7. Electrochemical heavy metal ion sensing was conducted on three different similar size marble to show repeatability.

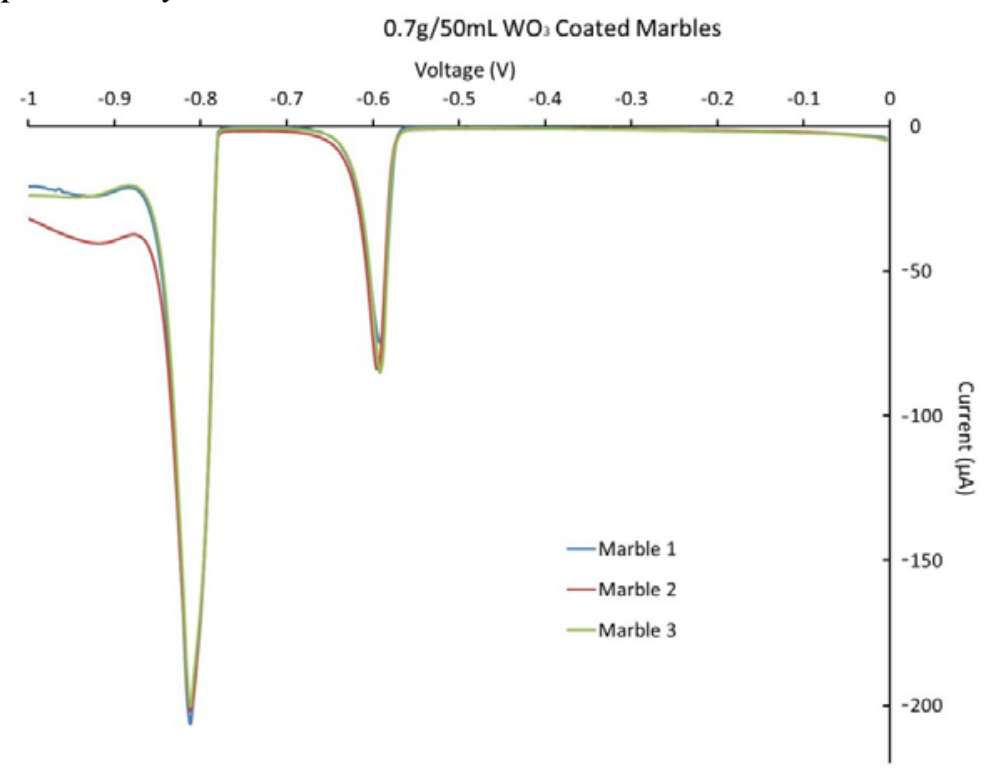

